\title{
El uso de la investigación en la política educativa: el caso del estado de Chihuahua
}

\author{
Romelia Hinojosa Luján \\ Esteban García Hernández \\ Rosa Angélica Rodríguez Arias \\ Juan Carlos Esparza Reyes
}

Departamento de Investigación Dirección de Investigación y Desarrollo Educativo Secretaría de Educación, Cultura y Deporte del estado de Chihuahua

\section{Resumen}

T a elaboración de los Estados de Conocimiento en el 2010 y el Diagnóstico de la investigación educativa realizado por la Red de Investigadores del estado de Chihuahua (REDIECH) y la Secretaría de Educación, Cultura y Deporte (SECYD) en 2012, permitieron describir la situación de la investigación en la entidad con respecto a su producción, diseminación y uso. Estos estudios muestran que existe un distanciamiento entre la investigación y las decisiones de la política educativa, así como discrepancias entre las demandas de la SECyD y la producción científica.

En el presente estudio se documenta un proyecto de investigación-acción realizado por el Departamento de Investigación de la Dirección de Desarrollo Educativo, que tiene como propósitos: fomentar el uso de aportes de la investigación educativa en la política educativa y acercar las demandas de investigación educativa a quienes producen conocimiento científico.

En los hallazgos se identifica una enorme brecha en las condiciones que existen entre las instituciones que realizan investigación educativa y los diferentes niveles de acercamiento que las autoridades educativas han tenido hacia la investigación, además de conceptos y expectativas de investigadores y autoridades hacia la investigación educativa. También se establece una agenda de investigación educativa y la identificación de algunos núcleos en germinación que comienzan a gestar espacios de vinculación.

Palabras clave: investigación educativa, política educativa, vinculación 


\section{Presentación}

El Plan Sectorial de Educación 20112016 de la SECYD, establece como prioritario: "impulsar la investigación, la sistematización y el análisis de la información educativa" (65), "fortalecer la vinculación con los sectores productivo, público y privado para establecer convenios de colaboración" (96); "promover y fortalecer la investigación en materia de educación, deporte, recreación y formación en valores para el desarrollo de innovaciones educativas" (101).

Por otro lado, la investigación de la investigación educativa realizada a través del Departamento de Investigación de la Dirección de Desarrollo de la Secyd (2010), en colaboración con algunas Instituciones de Educación Superior y la Rediech (Martínez, 2012), puso de manifiesto la apremiante necesidad de establecer una agenda de investigación educativa que rescatara las principales demandas que la autoridad establece con la idea de acercar la investigación a su característica de pertinencia.

Estas investigaciones también resaltan la urgencia de fomentar el uso de la investigación entre quienes ostentan puestos claves en la estructura del sistema educativo estatal con la idea de que sus decisiones estén fundamentadas en el conocimiento fidedigno del hecho educativo.

Orientados por estas consideraciones normativas y diagnósticas, el equipo del Departamento de Investigación realizó un proyecto de intervención para promover el uso de la investigación educativa dentro de la toma de decisiones de la política educativa, así como el acercamiento del quehacer investigativo hacia las demandas regionales de la investigación.

Este trabajo pretende otorgar a la investigación educativa el status de pertinente y útil al quehacer de la política en educación para la resolución de los problemas educativos que afectan a nuestra entidad.

Para realizar el proyecto se establecieron algunos parámetros teóricos y metodológicos. Para la fundamentación teórica se partió del enfoque de diálogo informado (Reimers y McGinn, 2000; Loera, 2002) a través de la cual plantean la necesidad de fomentar el uso de la investigación educativa en la toma de decisiones de la política pública a través de espacios en los que se fomente el diálogo constructivo.

La metodología es una amalgama de técnicas y métodos que responde a las necesidades de transformación de las prácticas que tradicionalmente rodeaban el quehacer de la investigación y de la política educativa; no responde de manera pura a un solo método o forma de realizar investigación. Se llevó a cabo un proyecto de intervención con enfoque participativo y se introdujeron una serie de estrategias para su seguimiento, sistematización y evaluación. Esas estrategias fueron: la observación y registro a través de diario de campo realizada por el equipo coordinador, la videofilmación, la audiograbación y el registro de algunas actividades llevado a cabo por los participantes del proyecto. Esta información se analizó y se sistematizó a través de matrices y redes mediante un procesamiento inductivo que llevó a la construcción de categorías que se describirán en el cuerpo de este documento. 
Cabe hacer mención que la entidad se dividió en tres regiones geográficas para el trabajo del proyecto: centro, norte y sur. La ciudad de Chihuahua fue sede de los trabajos de la región centro, $\mathrm{Cd}$. Juárez en el norte e Hidalgo del Parral en el sur. Se buscó el fortalecimiento de la descentralización de las acciones e instituciones.

Durante el desarrollo de este trabajo, se llevaron a cabo reuniones donde los participantes tuvieron la oportunidad de conocer de manera directa qué hacen y qué necesitan para propiciar la vinculación entre investigadores y tomadores de decisiones.

Primeramente, se organizó el plan general contemplando cuatro momentos dentro de la primera etapa del proyecto general. Esta ponencia abarca únicamente la primera etapa del proyecto, en estos momentos se encuentra en acción la segunda etapa, que será motivo de análisis en otro artículo. El proyecto no termina en el segundo acercamiento entre estas dos esferas de acción, sino que se plantea, al igual que el proceso de investigación-acción, una espiral sucesiva de acercamientos y acciones para resolver, superar o trabajar en torno a la problemática del poco uso que se le da a la investigación educativa en la toma de decisiones de la política educativa.

Esta primera etapa se resume en las siguientes acciones, números y participantes. Siete reuniones regionales: tres en el centro, dos en la norte y dos en la sur; más de una veintena de instituciones de Educación Superior (IES) participantes que se enuncian en la Tabla 1.

\begin{tabular}{|c|c|}
\hline Escuela Normal Rural "Ricardo Flores Magón" & Escuela Normal Superior del Edo. de Chihuahua "José E. Medrano" \\
\hline $\begin{array}{l}\text { Institución Benemérita y Centenaria Escuela Normal del } \\
\text { Estado de Chihuahua “Profr. Luis Urías Belderráin" }\end{array}$ & Instituto Tecnológico de Chihuahua II \\
\hline Red de Investigadores Educativos Chihuahua, A.C. & $\begin{array}{l}\text { Escuela de Trabajo Social del Estado de Chihuahua "Profra. y T.S. Gua- } \\
\text { dalupe Sánchez de Araiza" }\end{array}$ \\
\hline Universidad Autónoma de Chihuahua & Universidad Autónoma de Ciudad Juárez \\
\hline Universidad Tecnológica de Paquimé & Universidad Tecnológica Paso del Norte \\
\hline Colegio de Chihuahua & Instituto Tecnológico de Ciudad Juárez \\
\hline Escuela Normal Experimental “Miguel Hidalgo” & Instituto Tecnológico de Jiménez \\
\hline Centro de Investigación y Docencia & Centro de Estudios de Posgrado y Capacitación Mundo Nuevo \\
\hline Escuela de Antropología e Historia del Norte de México & Universidad Tecnológica de la Babícora \\
\hline $\begin{array}{l}\text { Centro de Actualización del Magisterio } \\
\text { Unidad Chihuahua y Unidad Cd. Juárez }\end{array}$ & $\begin{array}{l}\text { Centro Chihuahuense de Estudios de Posgrado. } \\
\text { Unidad Chihuahua y Unidad Cd. Juárez }\end{array}$ \\
\hline Universidad Tecnológica de Chihuahua & Universidad Tecnológica de Ciudad Juárez \\
\hline Centro de Investigación en Materiales Avanzados & Universidad Pedagógica Nacional del Estado de Chihuahua \\
\hline Universidad Politécnica Nacional & Universidad Tecnológica Junta de los Ríos \\
\hline Instituto Tecnológico de Chihuahua & Universidad Tecnológica Camargo \\
\hline Universidad Tecnológica de la Tarahumara & Universidad Tecnológica Paso del Norte \\
\hline Instituto Tecnológico de Hidalgo del Parral & Universidad Tecnológica de Chihuahua Unidad Ojinaga \\
\hline Universidad Tecnológica de Paquimé & Colegio de Doctores en Ciencias \\
\hline $\begin{array}{l}\text { Departamento de Investigación Educativa de la Direc- } \\
\text { ción de Educación Media y Terminal }\end{array}$ & $\begin{array}{l}\text { Programa de Investigación Educativa de la Dirección de Educación } \\
\text { Primaria }\end{array}$ \\
\hline
\end{tabular}


REVISTA DE INVESTIGACIÓN EDUCATIVA DE LA REDIECH N. 9

ISSN: 2007-4336

Por parte de las autoridades educativas se contó con la presencia de 396 personas: 205 mujeres y 191 hombres, distribuidas por región y por sexo como se presenta en la Gráfica 1.

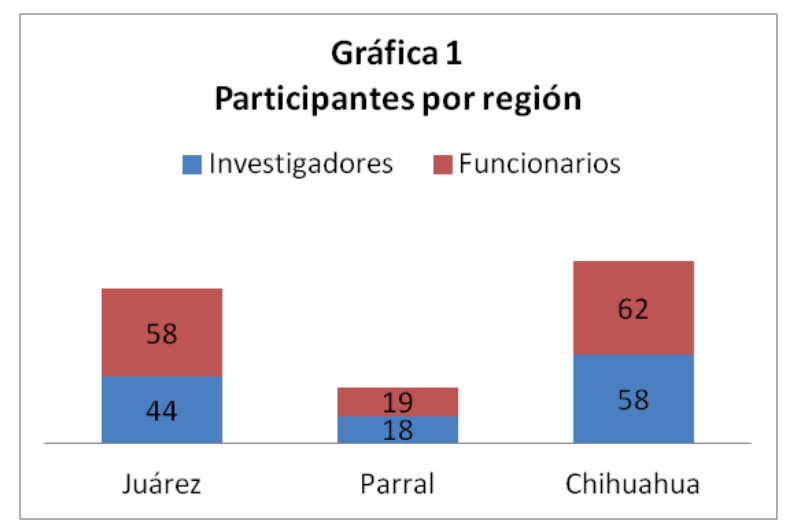

TABLA 2

Instancias Participantes

SECYD EDUCACIÓN BÁSICA

SeRVicios educativos del estado DE ChIHUAHUA

DEPARTAMENTO DE INVESTIGACIÓN EDUCATIVA DE LOS SERVICIOS EDUCATIVOS DEL ESTADO DE CHIHUAHUA

DiRECCIÓN DE DESARROLLO EDUCATIVO DE LA SECYD

DIRECCIÓN DE EDUCACIÓN PRIMARIA DE LOS SEECH

EDUC. ESPECIAL SEECH

PRogRama ESCUELAS DE TIEMPO COMPLETO

OfICINA ACADEMICA DE TELESECUNDARIAS

Programa ABRIENDO ESCUELAS PARA LA EQUIDAD

ICHIFE (INSTITUTO CHIHUAHUENSE DE INFRAESTRUCTURA FÍSICA EDUCATIVA)

DePto. Académico de PRIMARIA

DIRECCIÓN DE EDUCACIÓN ELEMENTAL

DEPARTAMENTO DE EDUCACIÓN PRIMARIA SECYD

INSTANCIA ESTATAL DE FORMACIÓN CONTINUA

Programa estatal de LeCtura

DEPARTAMENTO DE EDUCACIÓN SECUNDARIA SECYD

DePto. Educación FISICA y ARTES DE LA SECYD

PROGRAMA DE FORTALECIMIENTO DE LA EDUCACIÓN ESPECIAL Y DE LA INTEGRACIÓN EDUCATIVA

COLEGIO DE ESTUDIOS CIENTíFICOS Y TECNOLÓGICOS DEL ESTADO DE CHIHUAHUA

DePARTAMENTO DE EDUCACIÓN ESPECIAL SECYD

REFORMA PRIMARIA

Programa escuelas de CALIDAD
Los funcionarios que participaron fueron de los subsistemas estatal, federalizado y de algunos organismos descentralizados. En la Tabla 2 aparece el listado exhaustivo de las dependencias, unidades, programas, departamentos y direcciones de los niveles elementales, básicos, media superior y superior que participaron en las reuniones.

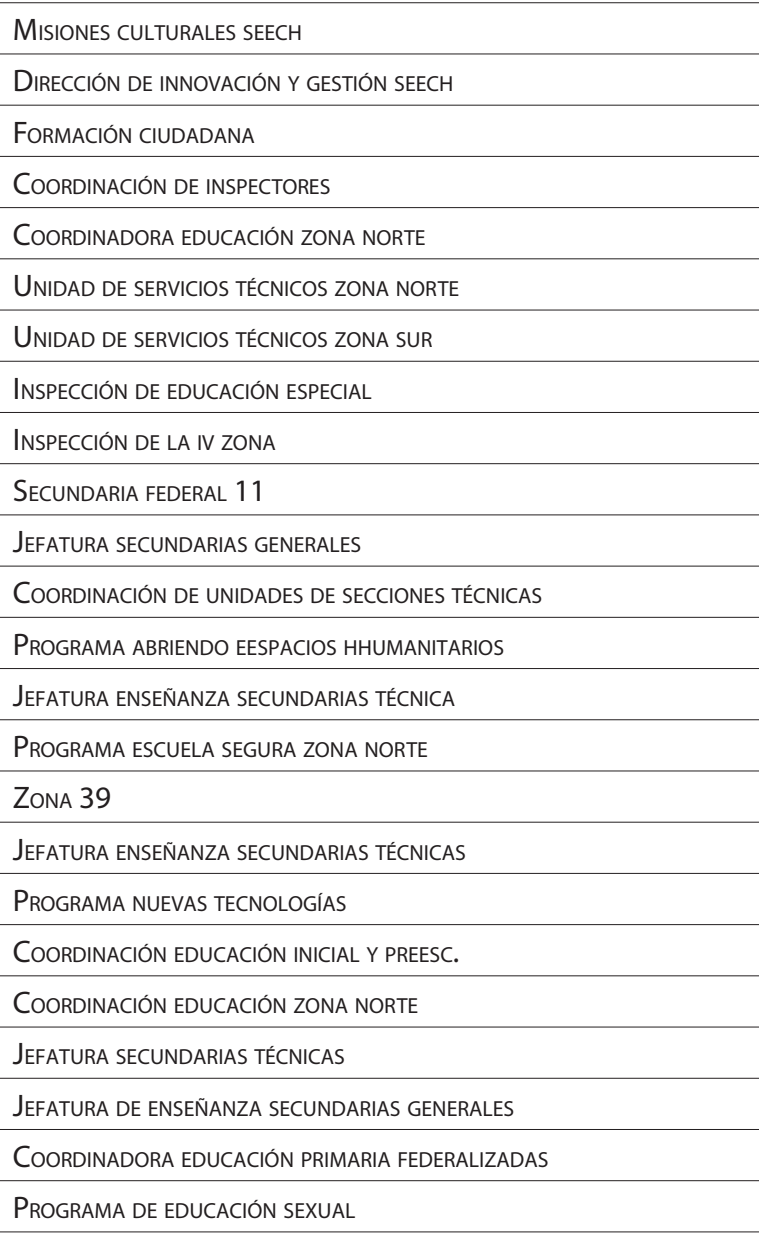


El uso de la investigación en la política educativa: el Caso del estado de Chihuahua

\begin{tabular}{|l|}
\hline PESE \\
\hline PROGRAMA DE PROYECTOS ACADEMICOS \\
\hline COORDINADORA DE PREESCOLAR ZONA NORTE \\
\hline COORDINADORA DE EDUCACIÓN ESPECIAL ZONA NORTE \\
\hline SUBSECRETARÍA ZONA NORTE \\
\hline COORDINACIÓN UNIDAD DE SERVICIOS TÉCNICOS ZONA SUR \\
\hline CONALEP (HIDALGO DEL PARRAL) \\
\hline INSP. ZONA 84 \\
\hline ESCUELA PREPARATORIA LÓPEZ VELARDE \\
\hline PRIMARIA ESTATAL 2357 \\
\hline INSTITUTO CERVANTES \\
\hline DEPARTAMENTO DE NORMALES DE LOS SEECH \\
\hline DIRECCIÓN DE EDUCACIÓN MEDIA Y TERMINAL DE LOS SEECH \\
\hline DIRECCIÓN GENERAL DE EDUCACIÓN TECNICA AGROPECUARIA \\
\hline USTEE \\
\hline SECUNDAIRA ESTATAL 3O66 \\
\hline DEPARTAMENTO DE EDUC. MEDIA SUPERIOR \\
\hline SUBSISTEMA DE PREPARATORIA ABIERTA \\
\hline JEFATURA SECUNDARIAS FEDERALES \\
\hline PROGRAMA DE CAPACITACIÓN AL MAGISTERIO PARA PREVENIR LA VIOLENCIA \\
CONTRA LAS MUJERES \\
\hline OFICINA ACADÉMICA EDUCACIÓN ESPECIAL \\
\hline
\end{tabular}

En el segundo momento de esta primera etapa, participaron investigadores y autoridades educativas por separado, lo cual se consideró necesario para el proyecto y la investigación. En el caso de los investigadores fue para la recuperación de experiencias de investigación educativa de diversas instituciones, sus condiciones de producción y divulgación. Por otro lado, en torno de las autoridades fue para la identificación de demandas específicas de Investigación Educativa (IE) y el acercamiento práctico a la experiencia investigativa. Sobre este momento se va a ahondar en la información ofrecida por ellos y ellas, a través del proceso de sistematización que realizó el equipo de investigación. Ésta se ofrecerá de manera breve en los apartados: "Condiciones generales de la IE" y "Funcionalidad

\begin{tabular}{|l|}
\hline PROGRAMA ESTATAL DE EDUCACIÓN PARA LA PREVENCIÓN DEL SIDA \\
\hline REFORMA SECUNDARIA \\
\hline $\begin{array}{l}\text { CONALEP (COLEGIO NACIONAL DE EDUCACIÓN PROFESIONAL TÉCNICA) } \\
\text { CHIH. }\end{array}$ \\
\hline COORDINACIÓN ESTATAL DE ASESORÍA Y SEGUIMIENTO \\
\hline DEPARTAMENTO DE EDUCACIÓN ELEMENTAL \\
\hline DEPARTAMENTO DE FORMADORAS Y ACTUALIZADORAS DE DOCENTES \\
\hline DEPARTAMENTO DE EDUCACIÓN MEDIA SUPERIOR \\
\hline PROGRAMA PARA ABATIR EL REZAGO EDUCATIVO EN EDUCACIÓN INICIAL Y \\
BÁSICA \\
\hline DEPTO. DE EDUCACIÓN ELEMENTAL \\
\hline ACC. COMPENSATORIAS \\
\hline SECUNDARIAS GENERALES SEECH \\
\hline SUBSISTEMA DE PREPARATORIA ABIERTA \\
\hline DGETI (DIRECCIÓN GENERAL DEL EDUCACIÓN TÉCNICA INDUSTRIAL) \\
\hline ADMINISTRATIVO DE LA DIRECCIÓN DE DESARROLLO EDUCATIVO \\
\hline APOYO ACADÉMICO DIRECCIÓN DE DESARROLLO EDUCATIVO \\
\hline COLEGIO DE BACHILLERES DEL ESTADO DE CHIHUAHUA \\
\hline CEDART (COLEGIO DE ARTES Y HUMANIDADES "DAVID ALFARO SIQUEIROS" \\
\hline COORDINACIÓN DE CECATIS (CENTROS DE CAPACITACIÓN PARA EL TRABAJO \\
INDUSTRIAL) \\
\hline PROGRAMA ESTATAL PARA LA EDUCACIÓN DEL SIDA (PEEPSIDA) \\
\hline
\end{tabular}

que se asigna a la IE: la experiencia de los funcionarios".

En el tercer momento de esta primera fase del proyecto, se hizo la devolución de la información brindada por ambos "mundos con vidas separadas" al colectivo completo de investigadores y funcionarios. Se les presentaron las condiciones generales de la IE que privan en la entidad: líneas de generación y aplicación del conocimiento de cada una de las IES, cuerpos académicos que tienen concordancia con la IE, así como las demandas o necesidades de las autoridades de diferentes niveles sobre IE para su contexto de trabajo.

Éstas últimas fueron recuperadas en un documento con formato de convocatoria del Consejo Nacional de Ciencia y Tecno- 
logía (CONACYT) con la intención de enviarlas al Fondo Mixto (Fomix, ConACYTGobierno del Estado) con la instrucción del entonces Secretario de Educación, de que se incorporara como demanda específica de investigación educativa.

\section{Condiciones generales de la IE}

De acuerdo con la información presentada por los investigadores de las diferentes instituciones que participaron en las tres reuniones de vinculación, es preciso mencionar que una característica que aparece como constante es la gran diferencia que existe en las condiciones en las que se lleva a cabo la investigación educativa.

\section{Organización de acciones de investigación}

La forma de organización de los grupos de investigación que existen en las IES, se presenta diferenciada y de manera incipiente en unas; prevalece la formación de equipos de trabajo que se conforman para realizar alguna investigación específica; por otro lado, es posible identificar a aquellas instituciones en las cuales se han integrado cuerpos académicos que se distinguen por trabajar bajo una línea, con metas definidas.

\section{Características de los investigadores}

En lo referente a los encargados de realizar investigación educativa, se identifican declaraciones en las que los grupos que se encargan de esta actividad, no cuentan con formación o experiencia en investigación.

En este aspecto se menciona: "Algunos profesores no participan en investigación por limitaciones en formación, aunque imparten materias relacionadas directamente con investigación, les falta capacitación para llevarla a cabo, no logran un nivel de formación en investigación en pregrado", además se tiene la percepción de que la investigación es un asunto de élite con distancia de las prácticas reales. De manera polarizada se ubican instituciones con profesionales que pertenecen al Sistema $\mathrm{Na}$ cional de Investigadores.

\section{Apoyos económicos y materiales para tareas de investigación}

Una constante que manifiestan los representantes de las IES se relaciona con la necesidad de recursos económicos y materiales para realizar investigación. Hay quien menciona que: "La investigación se hace a partir de la suma de buenas voluntades" o "El financiamiento sale del esfuerzo propio de las y los investigadores, de los propios estudiantes...", sin embargo, los recursos con los que cuentan, se presentan de manera diferenciada, pues los representantes de algunas instituciones plantearon que tienen la infraestructura física necesaria y reciben todos los recursos económicos para realizar investigación, además cuentan con apoyo para emprender acciones paralelas, tales como asistencia a cursos y congresos estatales, nacionales e incluso internacionales. Al interior de las instituciones que cuentan con apoyos, hay quien considera que estos no se distribuyen de manera equitativa al afirmar que "...algunos proyectos de la institución tienen muchos recursos, otros no". 


\section{Producción de investigación educativa}

Nuevamente en lo que se refiere a la producción de investigación educativa, se presentan grupos polarizados. En el primero, se establece que la investigación se basa en la producción de trabajos de tesis como opción de titulación de las diferentes licenciaturas y/o maestrías que se ofertan. En una de las instituciones se menciona: "La investigación no se lleva a cabo como tal, ésta se realiza en trabajos de titulación, en la cotidianeidad, en clase con ejercicios académicos, academias o colegiados, seminario de titulación, prácticas y servicio social, asesorías..." Por otra parte se identifican las instituciones que de manera paralela a la producción de tesis, llevan a cabo investigaciones que aportan a las necesidades de contar con conocimiento relativo a distintas áreas de conocimiento.

\section{Relación entre instituciones}

En la mayoría de los casos se plantea que la investigación educativa se lleva a cabo al interior de la institución, sin embargo, destacan algunos casos en los cuales se trabaja en coordinación con otras instituciones. Al respecto se menciona que "...la investigación educativa se condiciona por la falta de relación entre las instituciones que hacen investigación", sin embargo en algunas instituciones se plantea que se han establecido vínculos para trabajar con organizaciones no gubernamentales y con instituciones nacionales y extranjeras. Incluso en una institución específica se menciona “...hay una red que funciona para dar respuesta a las necesidades de la comunidad juarense y en comunicación con otros países que manejan la temática de la investigación en educación especial".

Con relación al tema de vinculación, en las declaraciones de los investigadores se identificaron opiniones que plantean la posibilidad que existe de que instituciones de educación superior se apoyen no únicamente en tareas de investigación, sino de profesionalización docente en diferentes ámbitos, en un caso específico se menciona la necesidad de "...generar redes de investigadores y participantes de la investigación para retroalimentación de todos los agentes involucrados (investigadores, beneficiarios y autoridades)", también se plantea como alternativa de vinculación: "...fomentar el intercambio de experiencias y encuentros sobre metodologías y procedimientos en las investigaciones". Otra alternativa hace referencia a la posibilidad de efectuar proyectos macro que se deriven de intereses comunes en torno a una temática, en donde se maneje una metodología y referentes teóricos comunes.

\section{Acciones de diseminación}

En torno a las prácticas de difusión y divulgación de la investigación educativa, la polaridad se presenta entre las instituciones que únicamente presentan los trabajos de tesis de sus egresados en eventos internos o ante otras instituciones, mientras que en otros casos se hace alusión a procesos amplios de difusión y divulgación con grupos de interés identificados. En relación a este aspecto hay quien concluye que "...no se capitalizan los productos de los esfuerzos que se realizan para efectuar investigación educativa". 
Funcionalidad que se asigna a la investigación educativa: la experiencia de los funcionarios.

\section{Decisiones individuales o colectivas}

De manera inicial, en las declaraciones realizadas por las autoridades educativas se perciben dos formas de tomar decisiones, un grupo mayoritario que desde su función decide de manera individual y otro que plantea un trabajo grupal en reuniones exprofeso como acción previa a la toma de decisiones. En este grupo se explica que las decisiones son tomadas de manera colectiva, a partir de consensos y de discusiones, que incluso pueden realizarse entre autoridades de diferente nivel jerárquico. Como ejemplos de estas declaraciones se mencionan las siguientes:

(Decidimos) a partir del trabajo colegiado, con base en el análisis de los diferentes temas $y / o$ problemas que correspondan a nuestro cargo (técnico-pedagógico).

En reflexiones que hacemos en mi equipo sobre la manera que puede repercutir o llevar como consecuencia la decisión tomada, con base al tema o problemática señalada, revisando de manera ampliada todas las posibilidades de solución a las necesidades observadas en las escuelas.

\section{Bases para la toma de decisiones}

En las declaraciones que hacen las autoridades educativas, con relación a las bases que consideran para la toma de decisiones se definen tres grandes grupos de respuestas: el primero se refiere a fuentes externas que los guían (normativos y órdenes), el se- gundo agrupa sus conocimientos propios con diferentes fuentes que van desde sus experiencias hasta aspectos más intuitivos, finalmente en el tercer grupo se ubica la información obtenida a partir de los contextos sobre los cuales se decide y en algunos casos de aportaciones derivadas de procesos de investigación.

\section{Fuentes externas}

En lo referente a las normas, reglamentos y lineamientos oficiales que guían las decisiones que se toman, las autoridades educativas mencionan las siguientes, que como es posible observar, van desde normas generales para el sistema educativo nacional como el Artículo $3^{\circ}$, hasta aquellos que son específicos a un departamento o programa, como los reglamentos internos. Algunas de las autoridades que declararon responder a aspectos normativos y considerar pocas posibilidades de hacer uso de resultados de investigación educativa hacen afirmaciones como la siguiente: "La toma de decisiones no queda en nuestras manos, tenemos que aplicar la política educativa".

- Artículo Tercero Constitucional

- Ley General de Educación

- Planes y programas de estudio de los diferentes niveles educativos

- Libros para el maestro

- Acuerdos oficiales, como el 592, el 593, el 648.

- Reglamento interior de trabajadores de la SEP 
Normativas y manuales que rigen la operatividad de programas federales y estatales que funcionan en la entidad.

- En la misión, visión, objetivos del programa.

- Lineamientos de la estructura educativa, como el Plan estratégico de las coordinaciones de las regiones, zonas de la entidad o de manera más específica los planes de trabajo de las diferentes dependencias.

En cuanto a las demandas que reciben, en este grupo se definen acciones que se deben realizar por mandatos de autoridades de nivel superior, estas se presentan de manera vertical y los informantes plantean que tienen las siguientes características:

- Pueden estar en la agenda y responder a un plan de trabajo o surgir sin una previa programación.

- Deben tener respuesta de manera inmediata, por su carácter emergente.

- Son indicaciones precisas de carácter operativo.

- Están asociadas a las problemáticas y necesidades de contextos específicos.

\section{Conocimientos personales}

En este grupo, las autoridades mencionan que sus decisiones se derivan básicamente de sus experiencias y los conocimientos con que cuentan. Hay quienes comentan: "Me baso en la experiencia y el conocimiento que he adquirido en el trayecto formativo de nuestra profesión" o "Podemos decir que (la toma de decisio- nes) es un proceso empírico ya que se toman en cuenta las experiencias vividas en cada región y en cada situación".

Aunque también hay quienes mencionan que hacen uso de su sentido común: “...y realizo adecuaciones cotidianas según necesidades y problemáticas en los centros escolares, basadas en el sentido común", también hay quien afirma: "Considero la percepción que tengo de los hechos", a lo que añade que esta acción la considera subjetiva.

En este grupo se ubican también las aseveraciones que hacen referencia al conocimiento inmediato que se tiene de las condiciones de los contextos en los cuales se van a definir acciones, con énfasis en las necesidades y demandas de los sujetos. Ejemplos de esto son los siguientes:

Las decisiones que se toman, consideran las características del centro educativo (...) incluso de la infraestructura.

Con las consideraciones y opiniones de los padres.

(Se toman en cuenta) grupos vulnerables, género, inclusión educativa, educación indígena, patrimonio cultural, violencia escolar.

En este apartado se ubican las declaraciones sobre la importancia de considerar los recursos humanos con los que se cuenta, pero principalmente los financieros, asociados a las reglas explícitas e implícitas para utilizarlos.

\section{Información derivada de procesos de investigación}

En este grupo se ubican las afirmacio- 
nes que plantean que las decisiones tienen como una de sus bases, indagaciones realizadas exprofeso para fundamentarlas y en menor medida la recuperación de información derivada de investigación educativa.

Las autoridades que mencionan que para decidir en su ámbito, consideran información referida a los sujetos y contextos que se van a involucrar o ver afectados por sus decisiones, hacen mención de una amplia variedad de experiencias de indagación que presentan diferente nivel en la sistematicidad y profundidad.

Las autoridades plantean que llevan a cabo observaciones libres, elaboran inventarios, aplican encuestas y cuestionarios para obtener información de aspectos precisos y analizan resultados de pruebas estandarizadas con el propósito de establecer estrategias de atención.

Además señalan que participan y promueven procesos de diagnóstico, autoevaluación y evaluación a partir de los cuales planifican acciones y valoran de manera periódica los servicios que ofrecen. Como ejemplos se recupera lo siguiente:

Para dar la capacitación a los docentes tenemos que investigar el universo a atender, en algunos casos tenemos que depurar las bases de datos, seleccionar a las escuelas focalizadas o al personal idóneo para recibir la capacitación los recursos económicos disponibles, el personal académico del que disponemos, los tiempos en que deben desarrollarse los trabajos, ya que hay fechas límite para cumplir con las capacitaciones.

(Valoramos) básicamente a partir de los documentos que evidencian el proceso aplicado con los beneficiarios a partir de reportes, así como con las observaciones planteadas.

Finalmente, en algunos casos específicos las decisiones que se toman se ven influenciadas por acciones de monitoreo y seguimiento, de los que destaca el acompañamiento técnico-pedagógico que se realiza con base en indicadores educativos, para identificar y dar atención a necesidades detectadas. En uno de los casos se menciona:

En el acompañamiento se realiza observación directa al desempeño de los docentes y a los aprendizajes de los alumnos, esto permite detectar los aciertos de los docentes, además los errores que son "áreas de oportunidades", se analizan los procesos de construcción del aprendizaje de los alumnos.

Un pequeño grupo de autoridades enfatizó la toma de sus decisiones basada en la información que aporta la investigación educativa. Mencionan de manera general que hacen uso de aportes teóricos: "La investigación sirve para fortalecer la toma de decisiones a las autoridades educativas", también identifican de manera específica estudios referidos a su ámbito de acción: "Me baso en la problemática que reflejan las estadísticas de infecciones de transmisión sexual y embarazo no planeado, proveniente de estudios de la Secretaría de Salud".

Hay quien explica por qué hace poco uso de referentes derivados de investigación educativa y menciona: "En muy bajo porcentaje me sustento (al tomar decisiones) en estudios o análisis porque no existen de manera suficiente, he aquí la importancia de fomentar la investigación educativa”. 


\section{Conclusiones}

De las experiencias obtenidas que se muestran en el cuerpo del trabajo y de los productos importantes que han quedado de la primera etapa del proyecto de vinculación se destacan las lecciones aprendidas a través de las acciones que se vienen realizando y muestran la incidencia en el uso de la investigación educativa para la toma de decisiones en política educativa. Merecen una explicación puntual de cada uno de ellos, misma que será motivo de otro documento amplio que por cuestiones de espacio en este trabajo no se podrá realizar. Se cerrará con un recuento breve de los mismos:

Promoción de condiciones propicias para el trabajo interfuncional, interinstitucional e intradisciplinar. Los funcionarios se sensibilizan ante la necesidad que se tiene del conocimiento científico en la toma de decisiones y los actores establecen como prioritario la divulgación de sus descubrimientos hacia la política educativa estatal. Se rompe la condición de "impermeabilidad" que había estado presente en las esferas participantes del proyecto.

Otorgamiento de voz y posicionamiento a las necesidades que el Sistema Educativo Estatal tiene sobre la IE. Las demandas de investigación educativa fueron enviadas por la Secretaría de Educación Cultura y Deporte al Conacyt y al Consejo Estatal de Ciencia y Tecnología del Estado de Chihuahua. Este es un ejercicio inédito en el sector, de tal manera que se espera impacte en un futuro inmediato en el lanzamiento de una convocatoria por parte de FomIX, que se acerque o ajuste a lo establecido por los participantes en este proyecto. El futuro es incierto, pero se trabaja abonando insumos que favorezcan las probabilidades en la ejecución de las acciones deseadas.

Generación de al menos dos núcleos en germinación (equipos interinstitucionales, intradisciplinarios e interfuncionales) que trabajan de manera más profunda la vinculación IE-Política Educativa en temáticas específicas: las reformas educativas y la condición de multiculturalidad en la educación.

\section{Bibliografía}

Loera, A. (2002). Los diálogos informados como método de concertación social. Teoría, Metodología y Prácticas Ejemplares de Concertación para el Desarrollo de América. Washington DC: INDES/BID.

Martínez, R. (2012). Diagnóstico de la Investigación Educativa en Chihuahua. Chihuahua, Chihuahua, México: Doble Hélice Editores.

Reimers, F., y McGinn, N. (2000). Diálogo informado. El uso de la investigación para apoyar la formulación de políticas educativas alrededor del mundo. México, D.F., México: Centro de Estudios Educativos-USJAL.

SECYd (2010). Colección Estados de Conocimiento de la Investigación Educativa en Chihuahua. Chihuahua, Chihuahua, México: Imprenta Ochoa.

SECYd (2011). Programa Sectorial de Educación 2011 2016. Chihuahua, Chihuahua, México: Talleres Gráficos de Gobierno del Estado. 